\section{PWE-030 5 YEAR EXPERIENCE- SAFETY/EFFICACY OF MIROCAM CAPSULE ENDOSCOPY IN PATIENTS WITH CARDIAC IMPLANTABLE ELECTRONIC DEVICES}

${ }^{1}$ Esther Hawkes*, ${ }^{1}$ Praful Patel, ${ }^{1}$ Markus Gwiggner, ${ }^{2}$ Hang Phan, ${ }^{2}$ Florina Borca, ${ }^{1}$ Imdadur Rahman. 'University Hospital Southampton, NHS Foundation Trust, Southampton, UK; ${ }^{2}$ University of Southampton, NIHR Southampton Biomedical Research Centre, Southampton, UK

\subsection{6/gutjnl-2019-BSGAbstracts.317}

Introduction Cardiac Implantable electronic devices (CIEDs) are seen to be a relative contraindication to capsule endoscopy (CE), due to concern that interaction between the 2 devices may lead to technical failure or interference to each device. Consequently, CE is performed infrequently in this cohort and hence significant pathology may be missed. The MiroCam capsule (Intromedic, Seoul) does not use the standard radiofrequency transmission, but a mechanism known as human body communication (HBC), considered to be safer. Our aim was to assess the safety and efficacy of CE in this cohort.

Methods A retrospective analysis of a prospectively collected database of CE procedures between May 2014 and January 2019. All CIEDs were remotely checked on the day or by interrogation of log files post procedure for any technical disruption. All video footage from the CE was analysed to check for image degradation or signs of interference. The positive findings (PY), and those deemed to be diagnostic (DY) on capsule endoscopy were documented.

Results 1018 CE procedures were performed during the study period. 45 of these were performed in patients with CIEDs (28 pacemakers, 6 implantable cardiac monitors, 5 defibrillators, 2 Cardiac Resynchronisation Therapy Pacemakers (CRT-P) and 4 CRT Defibrillators (CRT-D). The median age was 68 years with $62 \%$ females. The main indications were occult $(n=21)$ and overt GI bleeding $(n=15)$. Log files from all the implantable devices showed no signs of technical disruption. Video footage from $\mathrm{CE}$ showed no image degradation apart from one case which coincided with the use of a Microwave oven. The PY was $36 / 45=80 \%$ v $332 / 641=65.9 \%(p<0.05)$ and DY was $24 / 45=53.3 \% \quad \mathrm{v} 372 / 973=38.2 \%$ respectively in the CIED group compared to the non-CIED group $(\mathrm{p}<0.05)$.

Conclusions CE with the MiroCam capsule has been shown to be safe within this large cohort of patients, with no incidences of any technical disruptions in patients with CIEDs. Considering the higher rate of positive and diagnostic findings in CIED patients, CE with the MiroCam capsule in patients with an CIEDs should not be considered a contraindication. Further studies are required to see if this translates to other capsule manufacturers.

\section{PWE-031 NO ASSOCIATION BETWEEN HLA-DQA1*05 AND - DQB1*02 GENE DOSAGE AND CLINICAL PHENOTYPE OF COELIAC DISEASE}

${ }^{1}$ Hugo Penny*, 'Michael Rees, ${ }^{2} J$ ohn Goodwin, ${ }^{2}$ Tim Key, ${ }^{1}$ David Sanders. ${ }^{1}$ Academic Unit of Gastroenterology, Royal Hallamshire Hospital, Sheffield, UK; ${ }^{2}$ Histocompatibilty and Immunogenetics Laboratory, NHS Blood and Transplant, Sheffield, UK

\subsection{6/gutjnl-2019-BSGAbstracts.318}

Introduction Coeliac disease is a common gluten-sensitive enteropathy. Disease susceptibility is strongly associated with specific Human Leukocyte Antigen (HLA)-DQA1 and -DQB1 loci. Individuals with the HLA-DQA1*05:HLA-DQB1*02 heterodimer (referred to as HLA-DQ2.5) have the highest risk of developing coeliac disease, particularly if two copies of HLA-DQB $1 * 02$ are present. Understanding whether differences in the frequency of DQA1*05 and DQB1*02 accounts for differences in the clinical phenotype of coeliac disease is important for the development of personalised medicine for patients and was addressed in the following study.

Methods Demographic, clinical and laboratory data was retrospectively collected from adult patients attending the specialist coeliac disease clinic, Royal Hallamshire Hospital between 2008 to 2016 and correlated with the number of DQA $1 * 05$ : DQB1*02 combinations forming the DQ2.5 heterodimer (DQ2.5 gene dose), as well as the frequency of DQB1*02.

Results Four hundred and ninety patients had biopsy-proven coeliac disease and HLA genotype information. Individuals who were positive for the DQ2.5 heterodimer were more likely to be EMA positive than those who were DQ2.5 negative $(p=0.0132)$. There were no linear associations between the DQ2.5 gene dosage and clinical or laboratory parameters assessed. 190/490 (39\%) patients carried two copies of DQB1*02, 278/490 (57\%) patients had a single copy and DQB1*02 was absent in 22/490 (4\%) patients. The prevalence of folate deficiency was higher and mean haemoglobin levels lower, in individuals carrying two copies of DQB $1 \% 02$ than those with a single copy of DQB1*02 $(\mathrm{p}<0.001$ and $\mathrm{p}=0.002$, respectively), but neither were different in DQB1*02 negative individuals $(p>0.05)$. The carriage of two copies of DQB1*02 did not correlate with any other parameters.

Conclusions The presence of DQA1*05 and DQB1*02 is well documented to correlate with risk of developing coeliac disease. Our results suggest that there is no association between homozygosity/heterozygosity for DQA1*05 and DQB1*02 and the clinical phenotype of active disease. These results provide an important insight into the interpretation of HLA-DQ data in the setting of coeliac disease.

\section{PWE-032 CAN YOU DIAGNOSE ADULT COELIAC DISEASE WITHOUT THE NEED FOR A DUODENAL BIOPSY?}

Ravi Ranjan*, Helen Dallal, Cristina Dragos, Jodi Gaston-Ross. South Tees NHS Foundation Trust, Middlesbrough/Northallerton, UK

\subsection{6/gutjnl-2019-BSGAbstracts.319}

Introduction National Institute of Clinical Excellance (NICE) and British Society of Gastroenterology (BSG) guidelines advise that a Duodenal ( D2) Biopsy is required for a definitive diagnosis of coeliac disease. British Society of Paediatric Gastroenterology, Hepatology and Nutrition (BSPGHAN) 2013 guidelines indicate that this may not always be necessary. This study explored whether D2 Biopsy needs to be mandatory in the event of strongly positive IgA tissue transglutaminase (tTG).

Methods We extracted data from the pathology system for all adult patients who tested positive for IgA tTG between January to December 2018. For IgG and IgA tTG a fluorescent enzyme immunoassay technique is used ( Reagents from ThermoFisher on Phadia 250 analyser) by our local laboratory. tTG is reported as numeric value. Patients without D2 biopsies or biopsies more than 3 months apart from serology $(n=$ 147) and patients already known to have coeliac disease $(n=9)$ were excluded. D2 biopsy results were reviewed for all 